\title{
Flächen mit lauter Nabelpunkten
}

\author{
Arno Pauly \\ Arno Pauly studiert Informatik und Mathematik an der Fernuniversität Hagen und \\ Physik an der Albert-Ludwigs-Universität in Freiburg. Sein Informatikstudium hat er \\ im Jahr 2007 mit dem Master abgeschlossen.
}

Zweck des Folgenden ist ein kurzer Beweis des Satzes, dass jedes zweimal stetig differenzierbare Flächenstück im $\mathbb{R}^{3}$, welches nur aus Nabelpunkten besteht, in einer Ebene oder einer Sphäre enthalten ist. Das Resultat ist bekannt und war Gegenstand einer Arbeit von Philip Hartman [2]. Es hat aber nicht Eingang in die neuere Lehrbuchliteratur gefunden (vgl. [3], Problem auf S. 51), wohl weil der übliche kurze Beweis dreimalige stetige Differenzierbarkeit erfordert. Die Frage ist von einigem Interesse, da von ihr die Differenzierbarkeitsvoraussetzungen im geometrischen Beweis des Satzes von Liouville über konforme Abbildungen des dreidimensionalen Raumes abhängen.

\section{Differentialgeometrie der Flächen}

Wir erinnern zunächst an einige Grundbegriffe aus der Flächentheorie. Für Einzelheiten sei etwa auf [3] verwiesen. Im Anschluss an Gauß werden wir Flächen $M \subseteq \mathbb{R}^{3}$ als parametrisiert betrachten. Ein parametrisiertes Flächenstück der Klasse $C^{k}$ ist eine $k$-mal stetig differenzierbare Abbildung $f: U \rightarrow \mathbb{R}^{3}$, deren Differential $D f(u)$ an jeder Stelle $u \in U$ den Rang zwei hat. Dabei ist $U$ eine offene Teilmenge des $\mathbb{R}^{2}$, der Parameterbereich

Die Beschreibung von zweidimensionalen Flächen in dreidimensionalen Räumen gehört zu den originären Aufgaben der Differentialgeometrie. Eine wichtige Eigenschaft einer Fläche ist ihre Krümmung. Diese hängt prinzipiell sowohl von dem betrachteten Punkt als auch von der Richtung ab. In diesem Beitrag gibt der Autor einen elementaren Beweis des folgenden Satzes von P. Hartmann aus dem Jahr 1947, dass nämlich eine Fläche in der Ebene oder einer Sphäre enthalten sein muss, falls die Krümmung an jedem Punkt unabhängig von der Richtung ist. Im Unterschied zum wohlbekannten kurzen Beweis dieses Satzes, der dreimalige stetige Differenzierbarkeit erfordert, benötigt der Autor für seinen hier gegebenen elementaren Beweis nur die zweimalige stetige Differenzierbarkeit. 
von $f$. Wir werden im Folgenden $f$ als injektiv voraussetzen, so dass die Parameterwerte $u=\left(u_{1}, u_{2}\right)$ den Punkten $p \in M=f(U)$ eindeutig entsprechen.

Schreibt man für die partiellen Ableitungen $\partial / \partial u_{1}$ und $\partial / \partial u_{2}$ der Kürze halber $\partial_{1}$ und $\partial_{2}$, dann sind aufgrund der Rangbedingung die Vektoren $\partial_{1} f(u)$ und $\partial_{2} f(u)$ für jeden Parameterwert $u$ linear unabhängig und spannen die Tangentialebene $T_{p} M \subseteq \mathbb{R}^{3}$ der Fläche im Punkt $p=f(u)$ auf. Man erhält den (bis aufs Vorzeichen bestimmten) Einheitsnormalenvektor $v(u)$ der Fläche im Punkt $p$ als normiertes Vektorprodukt

$$
v(u)=\frac{\partial_{1} f \times \partial_{2} f}{\left\|\partial_{1} f \times \partial_{2} f\right\|}(u) .
$$

Die Abbildung $v: U \rightarrow \mathbb{R}^{3}$, die jedem Punkt $u \in U$ den Normalenvektor $v(u)$ zuordnet, heißt die Gaußabbildung von $f$.

Die Weingartenabbildung $L_{p}: T_{p} M \rightarrow T_{p} M$ der Fläche im Punkt $p=f(u)$ ist definiert als

$$
L_{p}=-\left(\left.D v\right|_{u}\right) \circ\left(\left.D f\right|_{u}\right)^{-1} \text {. }
$$

Sie ist ein selbstadjungierter Endomorphismus des zweidimensionalen Vektorraumes $T_{p} M \subseteq \mathbb{R}^{3}$. Ihre beiden Eigenwerte sind daher reell. Die Abbildung $L_{p}$ hat folgende geometrische Bedeutung: Ist $v \in T_{p} M$ ein Einheitstangentialvektor an die Fläche im Punkt $p$, dann ist das Skalarprodukt $\left\langle L_{p} v, v\right\rangle$ gleich der Krümmung des Normalschnittes der Fläche, den man erhält, wenn man $M$ mit der Ebene schneidet, die den Punkt $p$ enthält und in Richtung der Vektoren $v(u)$ und $v$ liegt.

Die verschiedenen Einheitsvektoren $v \in T_{p} M$ ergeben verschiedene Normalschnitte und damit im allgemeinen auch verschiedene Normalschnittkrümmungen. Als Hauptkrümmungen der Fläche im Punkt $p$ bezeichnet man die kleinste und die größte dieser Krümmungen. Es sind dies gerade die beiden Eigenwerte $\kappa_{1}(p)$ und $\kappa_{2}(p)$ von $L_{p}$. Damit die Weingartenabbildung und die Hauptkrümmungen definiert sind, ist $f$ als differenzierbar von der Klasse $C^{2}$ vorauszusetzen.

\section{Nabelpunkte}

Ein Punkt $p \in M$ heißt ein Nabelpunkt (oder Kreispunkt) von $M$, wenn $\kappa_{1}(p)=\kappa_{2}(p)$ gilt. Bezeichnet man diesen gemeinsamen Wert mit $\kappa(p)$, dann haben also alle Normalschnitte der Fläche im Punkt $p$ dieselbe Krümmung $\kappa(p)$, und die Weingartenabbildung ist ein Vielfaches der Identität: Es gilt $L_{p}=\kappa(p) I$, und damit, wenn man statt $\kappa(p)$ etwas ungenau $\kappa(u)$ schreibt,

$$
D v(u)=-\kappa(u) D f(u)
$$

Ziel der vorliegenden Arbeit ist nun ein Beweis des folgenden Satzes.

Satz. Sei $M=f(U)$ ein parametrisiertes Flächenstück der Klasse $C^{2}$ mit zusammenhängendem Parameterbereich $U$. Wenn jeder Punkt von $M$ ein Nabelpunkt ist, dann ist $M$ in einer Sphäre oder einer Ebene enthalten.

Flächen, die nur aus Nabelpunkten bestehen, werden gelegentlich als Nabelflächen (umbilical surfaces) bezeichnet. Der Satz besagt also, dass jede $C^{2}$-Nabelfläche in einer Sphäre oder einer Ebene enthalten ist. 
Sowohl der Satz als auch der im Folgenden gegebene Beweis lassen sich ohne weiteres auf den Fall einer $n$-dimensionalen Fläche der Klasse $C^{2}$ im $\mathbb{R}^{n+1}(n \geq 2)$ verallgemeinern.

Der wesentliche Punkt des Beweises besteht darin, zu zeigen, dass die Funktion $\kappa: u \mapsto$ $\kappa(u)$ auf $U$ konstant ist. Ist das nämlich der Fall, dann ergibt sich durch Integration der Gleichung (1)

$$
v=-\kappa f+c
$$

mit einem konstanten Vektor $c$. Falls $\kappa=0$, dann ist der Normalenvektor $v$ konstant. Man erhält für $j=1,2$

$$
\partial_{j}\langle f, v\rangle=\left\langle\partial_{j} f, v\right\rangle=0
$$

und damit die Ebenengleichung $\langle f, v\rangle=$ const. Ist aber $\kappa \neq 0$, dann folgt

$$
\left\|f-\frac{c}{\kappa}\right\|=\left\|\frac{v}{\kappa}\right\|=\frac{1}{|\kappa|}
$$

und das ist die Gleichung einer Sphäre. Es bleibt also nur die Konstanz von $\kappa$ zu beweisen. Setzt man voraus, dass das Flächenstück sogar differenzierbar von der Klasse $C^{3}$ ist, dann ergibt sich die Behauptung wie folgt: Gleichung (1) besagt

$$
\partial_{1} v=-\kappa \partial_{1} f, \quad \partial_{2} v=-\kappa \partial_{2} f .
$$

Wendet man $\partial_{2}$ auf die erste, $\partial_{1}$ auf die zweite dieser Gleichungen an und setzt mit $\partial_{1} \partial_{2} v=\partial_{2} \partial_{1} v$ die Resultate gleich, dann ergibt sich wegen der linearen Unabhängigkeit der Vektoren $\partial_{1} f$ und $\partial_{2} f$, dass $\partial_{1} \kappa=\partial_{2} \kappa=0$ ist. Also ist $\kappa$ konstant. Das Argument versagt, wenn $f$ nur von der Klasse $C^{2}$ ist.

\section{Beweis des Satzes}

Es genügt, zu zeigen, dass jeder Punkt $p_{0} \in M$ eine Umgebung in $M$ besitzt, die in einer Ebene oder einer Sphäre enthalten ist. Hinreichend kleine Umgebungen lassen sich nach Monge als Graph einer Funktion über der Tangentialebene in $p_{0}$ parametrisieren. Man kann daher annehmen, dass $f$ die Gestalt

$$
f\left(u_{1}, u_{2}\right)=\left(u_{1}, u_{2}, h\left(u_{1}, u_{2}\right)\right)
$$

hat mit einer zweimal stetig differenzierbaren reellen Funktion $h$. Dann ist

$$
D f=\left(\partial_{1} f, \partial_{2} f\right)=\left(\begin{array}{cc}
1 & 0 \\
0 & 1 \\
\partial_{1} h & \partial_{2} h
\end{array}\right)
$$

und die Gaußabbildung

$$
v=\frac{\partial_{1} f \times \partial_{2} f}{\left\|\partial_{1} f \times \partial_{2} f\right\|}=-\frac{1}{\gamma}\left(\partial_{1} h, \partial_{2} h, 1\right)
$$

mit $\gamma:=\sqrt{1+\left(\partial_{1} h\right)^{2}+\left(\partial_{2} h\right)^{2}}$. Setzt man diese Ausdrücke in die Matrixgleichung (1) ein und vergleicht entsprechende Matrixelemente, dann ergibt sich

$$
\partial_{1}\left(\partial_{1} h / \gamma\right)=\kappa, \quad \partial_{1}\left(\partial_{2} h / \gamma\right)=0, \quad \partial_{2}\left(\partial_{2} h / \gamma\right)=\kappa, \quad \partial_{2}\left(\partial_{1} h / \gamma\right)=0 .
$$


Die vierte dieser Gleichungen besagt, dass $\partial_{1} h / \gamma$ nicht von $u_{2}$ abhängt. Es ist also $\partial_{1} h / \gamma=a_{1}\left(u_{1}\right)$ mit einer stetig differenzierbaren Funktion $a_{1}$. Ebenso folgt aus der zweiten Gleichung $\partial_{2} h / \gamma=a_{2}\left(u_{2}\right)$. Die beiden übrigen Gleichungen liefern nun

$$
a_{1}^{\prime}\left(u_{1}\right)=a_{2}^{\prime}\left(u_{2}\right)=\kappa\left(u_{1}, u_{2}\right),
$$

und diese Gleichung zeigt, dass $\kappa$ weder von $u_{1}$ noch von $u_{2}$ abhängt, also konstant ist.

\section{Bemerkungen}

Der Satz geht zurück auf eine der ersten Arbeiten zur Differentialgeometrie der Flächen, Meusniers Mémoire sur la courbure des surfaces aus dem Jahre 1776. Das im dritten Abschnitt gegebene Argument findet sich in ähnlicher Form bei Monge ([4, XIX, S. 172175]), ist aber offenbar in Vergessenheit geraten. Die von Hartman [2] verwendete Methode beruht grob gesprochen darauf, dass es genügt, wenn Integrabilitätsbedingungen der Differentialgeometrie in einem integrierten Sinne erfüllt sind. Hartman hat diesen Gedanken in der Folge systematisch verwendet, um klassische Sätze der Differentialgeometrie unter schwächeren als den üblichen Differenzierbarkeitsbedingungen zu beweisen. Das Thema der Nabelflächen ist von einem anderen Standpunkt erneut aufgegriffen worden. Es geht dabei um die Frage der Stabilität der Aussage des Satzes, genauer darum, ob eine Fläche, deren sämtliche Punkte in einem zu präzisierenden Sinne beinahe Nabelpunkte sind, notwendig nahe bei einer Sphäre liegt. Positive Resultate in dieser Richtung finden sich bei Pogorelov ([5, S. 493]), Reshetnyak [6] und de Lellis und Müller [1]. Als Nebenresultat ergibt sich insbesondere, dass der Satz auch für Flächen der Sobolevklasse $W^{2,2}$ gilt.

Danksagung. Der Autor möchte sich herzlich bei Herrn Prof. Wolfgang Kühnel sowie dem anonymen Gutachter für ihre sehr hilfreichen Kommentare bedanken.

\section{Literatur}

[1] de Lellis, C.; Müller, S.: Optimal rigidity estimates for nearly umbilical surfaces. J. Differential Geom. 69 (2005), 75-110

[2] Hartman, P.: Systems of total differential equations and Liouville's theorem on conformal mappings. Amer. J. Math. 69 (1947), 327-332.

[3] Kühnel, W.: Differentialgeometrie. Vieweg Verlag, Braunschweig und Wiesbaden 2005.

[4] Monge, G.: Application de l'analyse à la géométrie. Bernard, Paris 1807.

[5] Pogorelov, A.V.: Extrinsic geometry of convex surfaces. Translations of Mathematical Monographs v. 35, American Mathematical Society 1973.

[6] Reshetnyak, Yu.G.: Some estimates for almost umbilical surfaces. Siberian Math. J. 9 (1968), 671-682.

Arno Pauly

Albert-Ludwigs-Universität Freiburg

In den Gässlewiesen 3

D-79111 Freiburg

e-mail: arno.pauly@saturn.uni-freiburg.de 